\title{
GAUSSIAN-TYPE QUADRATURE RULES FOR MÜNTZ SYSTEMS*
}

\author{
GRADIMIR V. MILOVANOVIĆ ${ }^{\dagger}$ AND ALEKSANDAR S. CVETKOVIĆ
}

\begin{abstract}
A method for constructing the generalized Gaussian quadrature rules for Müntz polynomials on $(0,1)$ is given. Such quadratures possess several properties of the classical Gaussian formulae (for polynomial systems), such as positivity of the weights, rapid convergence, etc. They can be applied to the wide class of functions, including smooth functions, as well as functions with end-point singularities, such as those in boundary-contact value problems, integral equations with singular kernels, complex analysis, potential theory, etc. The constructive method is based on an application of orthogonal Müntz polynomials introduced by Badalyan [Akad. Nauk Armyan. SSR. Izv. Fiz.-Mat. Estest. Tehn. Nauk, 8 (1955), pp. 1-28; 9 (1956), pp. 3-22 (Russian; Armenian summary)] and studied intensively by Borwein, Erdélyi, and Zhang [Trans. Amer. Math. Soc., 342 (1994), pp. 523-542], as well as by Milovanović on a numerical procedure for evaluation of such polynomials with high precision [Müntz orthogonal polynomials and their numerical evaluation, in Applications and Computation of Orthogonal Polynomials, Internat. Ser. Numer. Math. 131, W. Gautschi, G. H. Golub, and G. Opfer, eds., Birkhäuser, Basel, 1999, pp. 179-194]. The method is numerically stable. Some numerical examples are included.
\end{abstract}

Key words. numerical integration, generalized Gaussian quadrature, Müntz polynomials, zeros, nodes, weights

AMS subject classifications. 41A55, 65D30, 65D32

DOI. $10.1137 / 040621533$

1. Introduction. Gauss's famous method of approximate integration from 1814 can be extended in a natural way to nonpolynomial functions, taking a system of linearly independent functions

$$
\left\{P_{0}(x), P_{1}(x), P_{2}(x), \ldots\right\} \quad(x \in[a, b]),
$$

usually chosen to be complete in some suitable space of functions (cf. Gautschi [6], Karlin and Studden [12], Harris and Evans [11], Ma, Rokhlin, and Wandzura [13]). If $d \sigma(x)$ is a given nonnegative measure on $[a, b]$ and the quadrature rule

$$
\int_{a}^{b} f(x) d \sigma(x)=\sum_{k=1}^{n} A_{k} f\left(x_{k}\right)+R_{n}(f)
$$

is such that it integrates exactly the first $2 n$ functions in (1.1), we call rule (1.2) Gaussian with respect to the system (1.1). The existence and uniqueness of a Gaussian quadrature rule (1.2) with respect to the system (1.1), or, for brevity, a generalized Gaussian formula, is always guaranteed if the first $2 n$ functions of this system constitute a Chebyshev system on $[a, b]$. Then, all the weights $A_{1}, \ldots, A_{n}$ in (1.2) are positive. In terms of moment spaces, the Gaussian rule corresponds to the unique lower principal representation of the measure $d \sigma(x)$ (see Karlin and Studden [12]).

* Received by the editors December 26, 2004; accepted for publication (in revised form) May 10, 2005; published electronically November 22, 2005. This work was supported in part by the Swiss National Science Foundation (SCOPES Joint Research Project New Methods for Quadrature) and the Serbian Ministry of Science and Environmental Protection (Project Applied Orthogonal Systems, Constructive Approximation and Numerical Methods, grant 2002).

http://www.siam.org/journals/sisc/27-3/62153.html

${ }^{\dagger}$ Department of Mathematics, Faculty of Electronic Engineering, University of Niš, P. O. Box 73, 18000 Niš, Serbia (grade@ni.ac.yu, aca@elfak.ni.ac.yu). 
The generalized Gaussian quadratures for Müntz systems goes back to Stieltjes's paper [22] of 1884. Taking $P_{k}(x)=x^{\lambda_{k}}$ on $[a, b]=[0,1]$, where $0 \leq \lambda_{0}<\lambda_{1}<\cdots$, he showed the existence of Gaussian formulae. In his short note he considered also Gauss-Radau formulae.

A numerical algorithm for constructing the generalized Gaussian quadratures was recently investigated by Ma, Rokhlin, and Wandzura [13]. They take a Chebyshev system of functions $\left\{P_{0}, P_{1}, \ldots, P_{2 n-1}\right\}$ on $[a, b]$ with the following properties:

(1) $P_{k} \in C^{1}[a, b] \quad(k=0,1, \ldots, 2 n-1)$;

(2) the determinants

$$
\left|\begin{array}{cccccc}
P_{0}\left(x_{1}\right) & \cdots & P_{0}\left(x_{n}\right) & P_{0}^{\prime}\left(x_{1}\right) & \cdots & P_{0}^{\prime}\left(x_{n}\right) \\
P_{1}\left(x_{1}\right) & & P_{1}\left(x_{n}\right) & P_{1}^{\prime}\left(x_{1}\right) & & P_{1}^{\prime}\left(x_{n}\right) \\
\vdots & & & & & \\
P_{2 n-1}\left(x_{1}\right) & & P_{2 n-1}\left(x_{n}\right) & P_{2 n-1}^{\prime}\left(x_{1}\right) & & P_{2 n-1}^{\prime}\left(x_{n}\right)
\end{array}\right|
$$

are nonzero for any set of $n$ points $x_{1}, \ldots, x_{n} \in[a, b]\left(x_{i} \neq x_{j}\right.$ for $\left.i \neq j\right)$.

Such a system will be referred to as an extended Hermite (EH) system.

The procedure given in [13] requires the construction of the functions

$$
\xi_{i}(x)=\sum_{j=1}^{2 n} \alpha_{i j} P_{j-1}(x), \quad \eta_{i}(x)=\sum_{j=1}^{2 n} \beta_{i j} P_{j-1}(x) \quad(i=1, \ldots, n)
$$

such that

$$
\left\{\begin{array} { l } 
{ \xi _ { i } ( x _ { k } ) = 0 , } \\
{ \xi _ { i } ^ { \prime } ( x _ { k } ) = \delta _ { i k } , }
\end{array} \quad \left\{\begin{array}{l}
\eta_{i}\left(x_{k}\right)=\delta_{i k} \\
\eta_{i}^{\prime}\left(x_{k}\right)=0
\end{array}\right.\right.
$$

for all $i=1, \ldots, n$ and all $k=1, \ldots, n$. The algorithm is ill conditioned (see [13, Remark 6.2]). In order to obtain the double precision results (REAL*8), the authors performed the computations in extended precision (Q-arithmetic, i.e., REAL*16) for generating Gaussian quadratures up to order 20, and in MAтнемAтісA (120-digit operations) for generating Gaussian quadratures of higher orders $(n \leq 40)$. In particular, they considered the following important cases of EH systems:

$$
\left\{1, \log x, x, x \log x, \ldots, x^{n-1}, x^{n-1} \log x\right\}
$$

and

$$
\left\{1, x^{s}, x, x^{s+1}, \ldots, x^{n-1}, x^{s+n-1}\right\}
$$

for $s=1 / 3, s=-1 / 3, s=-2 / 3$. The case (1.5) was also considered by Andronov [1] in order to solve certain boundary-contact value problems.

For a given sequence of real numbers $\Lambda=\left\{\lambda_{0}, \lambda_{1}, \lambda_{2}, \ldots\right\}$ given in nondecreasing order, i.e., $\lambda_{k} \leq \lambda_{k+1}, k \in \mathbb{N}_{0}$, in this paper we consider Müntz polynomials as linear combinations of the Müntz system

$$
\left\{x^{\lambda_{0}}, x^{\lambda_{1}}, \ldots, x^{\lambda_{n}}\right\} .
$$

By $M_{n}(\Lambda)$ we denote the set of all such polynomials, i.e.,

$$
M_{n}(\Lambda)=\operatorname{span}\left\{x^{\lambda_{0}}, x^{\lambda_{1}}, \ldots, x^{\lambda_{n}}\right\},
$$

where the linear span is over the real numbers. 
Here, we present an alternatively numerical method for constructing the generalized Gaussian quadratures for Müntz polynomials

$$
\int_{0}^{1} f(x) d \sigma(x)=\sum_{k=1}^{n} A_{k} f\left(x_{k}\right)+R_{n}(f),
$$

which are exact for each $f \in M_{2 n-1}(\Lambda)$. Our method is rather stable and simpler than the previous one. It performs calculations in double precision arithmetic to get double precision results.

As is well known (see [14], [28]), the Gaussian quadrature rule is unique, provided the measure $\sigma$ has a nonnegative absolutely continuous part and has finitely many atoms on $[0,1]$. Such quadratures possess several properties of the classical Gaussian formulae (for polynomial systems), such as positivity of the weights, rapid convergence, etc. They can be applied to the wide class of functions, including smooth functions, as well as functions with end-point singularities, such as those in boundarycontact value problems (see [1]), integral equations, complex analysis, potential theory, and several other fields (see [13]).

This paper is organized as follows. Section 2 is devoted to some properties of orthogonal Müntz polynomials on $(0,1)$ and their connection with orthogonal rational functions, including numerical evaluation of such generalized polynomials. A numerical method for constructing the quadrature rules (1.7) is presented in section 3. Finally, in section 4 we give numerical results.

2. Orthogonal Müntz-Legendre polynomials. We are interested in the class of Müntz polynomials introduced by Badalyan [2], and we refer to it as MüntzLegendre polynomials.

Let a complex sequence $\Lambda=\left\{\lambda_{0}, \lambda_{1}, \lambda_{2}, \ldots\right\}$ be such that $\operatorname{Re}\left(\lambda_{k}\right)>-1 / 2$ for every $k \in \mathbb{N}_{0}$ and let $\Lambda_{n}=\left\{\lambda_{0}, \lambda_{1}, \ldots, \lambda_{n}\right\}$. If $\Gamma$ is a simple contour surrounding all the zeros of the denominator in the rational function

$$
W_{n}(s)=\prod_{k=0}^{n-1} \frac{s+\bar{\lambda}_{k}+1}{s-\lambda_{k}} \cdot \frac{1}{s-\lambda_{n}} \quad\left(n \in \mathbb{N}_{0}\right),
$$

then the Müntz-Legendre polynomials are defined by (see [2], [3], [4], [15], [25])

$$
P_{n}(x)=P_{n}\left(x ; \Lambda_{n}\right)=\frac{1}{2 \pi i} \oint_{\Gamma} W_{n}(s) x^{s} d s .
$$

In the case $n=0$, an empty product in (2.1) should be taken to be equal to 1 .

If $\lambda_{k} \neq \lambda_{j}(k \neq j)$, by the Cauchy residue theorem, these generalized polynomials can be expressed in a power form as

$$
P_{n}(x)=\sum_{k=0}^{n} C_{n k} x^{\lambda_{k}} .
$$

If there exists a pair with the property $\lambda_{k}=\lambda_{j}, k \neq j$, an application of Cauchy residue theorem produces terms with log function included. For example, we consider the important special case, where

$$
\lambda_{2 k}=\lambda_{2 k+1}=k \quad(k=0,1, \ldots) .
$$


Namely, we take $\lambda_{2 k}=k$ and $\lambda_{2 k+1}=k+\varepsilon(k=0,1, \ldots)$, where $\varepsilon$ decreases to zero. The corresponding limit process leads to orthogonal Müntz polynomials with logarithmic terms. Then, (2.1) becomes

$$
W_{n}(s)= \begin{cases}\prod_{\nu=0}^{m-1}\left(\frac{s+\nu+1}{s-\nu}\right)^{2} \frac{1}{s-m} & \text { when } n=2 m, \\ \prod_{\nu=0}^{m}\left(\frac{s+\nu+1}{s-\nu}\right)^{2} \frac{1}{s+m+1} & \text { when } n=2 m+1 .\end{cases}
$$

Applying the Cauchy residue theorem to the integral in (2.2), with this rational function, we obtain the following representation for the corresponding Müntz polynomials:

$$
P_{n}(x)=R_{n}(x)+S_{n}(x) \log x \quad(n=0,1, \ldots),
$$

where $R_{n}(x)$ and $S_{n}(x)$ are algebraic polynomials of degree [n/2] and [(n-1)/2], respectively, i.e.,

$$
R_{n}(x)=\sum_{\nu=0}^{[n / 2]} a_{\nu}^{(n)} x^{\nu}, \quad S_{n}(x)=\sum_{\nu=0}^{[(n-1) / 2]} b_{\nu}^{(n)} x^{\nu} .
$$

Notice that $P_{n}(1)=R_{n}(1)=1$. A general expression for the polynomial $P_{n}, n \in$ $\mathbb{N}_{0}$, was given in [17]. These Müntz polynomials can be used in the proof of the irrationality of $\zeta(3)$ and of other familiar numbers (see [4, pp. 372-381] and [27]).

In the special case when $\lambda_{0}=\lambda_{1}=\cdots=\lambda,(2.2)$ gives

$$
P_{n}\left(x ; \Lambda_{n}\right)=x^{\lambda} L_{n}(-(\lambda+\bar{\lambda}+1) \log x),
$$

where $L_{n}(x)$ is the Laguerre polynomial orthogonal with respect to $e^{-x}$ on $[0, \infty)$ and such that $L_{n}(0)=1$ (cf. [5, p. 145]).

TheOREM 2.1. For the Müntz-Legendre polynomials (2.2) the following orthogonality relation holds:

$$
\left(P_{n}, P_{m}\right)=\int_{0}^{1} P_{n}(x) \overline{P_{m}(x)} d x=\frac{\delta_{n m}}{\lambda_{n}+\bar{\lambda}_{n}+1} .
$$

According to this theorem we can construct orthonormal sequence of MüntzLegendre polynomials, denoted by $p_{n}(x):=p_{n}(x ; \Lambda)=\left(\lambda_{n}+\bar{\lambda}_{n}+1\right)^{1 / 2} P_{n}(x), n \in \mathbb{N}_{0}$.

For a general nondecreasing sequence $\Lambda$, the case when some of $\lambda_{k}, k \in \mathbb{N}_{0}$, are the same, it is complicated to express the basis functions in a closed form. For example, if all $\lambda_{k}, k \in \mathbb{N}_{0}$, are different, we have that the system of basis functions is given by $\left\{x^{\lambda_{0}}, x^{\lambda_{1}}, \ldots\right\}$. In the case $\lambda_{k}=\lambda_{k+1}=k, k \in \mathbb{N}_{0}$, we have that the system of basis functions is given by $\{1, \log x, x, x \log x, \ldots\}$. To make notation simple, we use the notation $x^{\Lambda}$ to denote the set of basis functions for the general nondecreasing sequence $\Lambda$. Also, we introduce the notation $x^{\Lambda_{n}}$ for the first $n$ functions in the system $x^{\Lambda}$.

Putting $\lambda_{k}+\beta / 2$ instead of $\lambda_{k}, k=0,1, \ldots$, in the sequence $\Lambda$, we can define a kind of Müntz-Jacobi polynomials $P_{n}^{(\beta)}(x)$ by

$$
P_{n}^{(\beta)}(x)=\frac{x^{-\beta / 2}}{2 \pi i} \oint_{\Gamma} W_{n}^{(\beta)}(s) x^{s} d s,
$$


where

$$
W_{n}^{(\beta)}(s)=\prod_{k=0}^{n-1} \frac{s+\bar{\lambda}_{k}+\beta / 2+1}{s-\lambda_{k}-\beta / 2} \cdot \frac{1}{s-\lambda_{n}-\beta / 2} .
$$

Then the following result holds.

Theorem 2.2. Let $\beta \in \mathbb{R}$ and $\operatorname{Re} \lambda_{k}>-(\beta+1) / 2$ for each $k \in \mathbb{N}_{0}$. Then

$$
\left(P_{n}^{(\beta)}, P_{m}^{(\beta)}\right)=\int_{0}^{1} P_{n}^{(\beta)}(x) \overline{P_{m}^{(\beta)}(x)} x^{\beta} d x=\frac{\delta_{n m}}{\lambda_{n}+\bar{\lambda}_{n}+\beta+1} .
$$

Let $P_{n}(\cdot ; \Lambda), n \in \mathbb{N}_{0}$, denote the Müntz-Legendre polynomials for a nondecreasing sequence $\Lambda=\left\{\lambda_{0}, \lambda_{1}, \lambda_{2}, \ldots\right\}$. Then it is very easy to verify that $P_{n}^{(\beta)}(x)=$ $x^{-\beta / 2} P_{n}(x ; \Lambda+\beta / 2)$, where $P_{n}(\cdot ; \Lambda+\beta / 2)$ are really Müntz-Legendre polynomials orthogonal with respect to the Legendre measure. This means that an orthogonality with respect to $x^{\beta}$ is obtained straightforwardly from Theorem 2.1 .

Also, some recurrence relations exist for Müntz-Legendre polynomials, e.g.,

$$
x P_{n}^{\prime}(x)-x P_{n-1}^{\prime}(x)=\lambda_{n} P_{n}(x)+\left(1+\bar{\lambda}_{n-1}\right) P_{n-1}(x)
$$

and

$$
P_{n}(x)=P_{n-1}(x)-\left(\lambda_{n}+\bar{\lambda}_{n-1}+1\right) x^{\lambda_{n}} \int_{x}^{1} t^{-\lambda_{n}-1} P_{n-1}(t) d t \quad(x \in(0,1]) .
$$

It is easy to prove that

$$
P_{n}(1)=1 \quad \text { and } \quad P_{n}^{\prime}(1)=\lambda_{n}+\sum_{k=0}^{n-1}\left(\lambda_{k}+\bar{\lambda}_{k}+1\right) .
$$

Taking $x=e^{-t}$, the Müntz-Legendre polynomials can be expressed in terms of a Laplace transform. Namely, we can prove the following (cf. [17]).

THEOREM 2.3. If $W_{n}(s)$ is given by (2.1) and

$$
G_{n}(s)=-W_{n}(-s)=\prod_{k=0}^{n-1} \frac{s-\left(\bar{\lambda}_{k}+1\right)}{s+\lambda_{k}} \cdot \frac{1}{s+\lambda_{n}},
$$

then $P_{n}\left(e^{-t}\right)$ is the inverse Laplace transform of $G_{n}(s)$, i.e.,

$$
P_{n}\left(e^{-t}\right)=\mathcal{L}^{-1}\left[G_{n}(s)\right] .
$$

In the proof of this result we can take, for example, $\alpha>1 / 2$, and then prove that

$$
P_{n}\left(e^{-t}\right)=\frac{1}{2 \pi i} \int_{\alpha-i \infty}^{\alpha+i \infty} G_{n}(s) e^{s t} d s .
$$

An interesting question is connected with the zero distribution of the MüntzLegendre polynomials for a real sequence $\Lambda$. A nice proof of the following result was given in [3].

TheOREM 2.4. For real numbers $\lambda_{\nu}>-1 / 2(\nu=0,1, \ldots)$ the Müntz-Legendre polynomial $P_{n}\left(x ; \Lambda_{n}\right)$ has exactly $n$ distinct zeros in $(0,1)$, and it changes sign at each of these zeros. Furthermore, the zeros of the polynomials

$$
P_{n-1}\left(x ; \Lambda_{n-1}\right) \quad \text { and } \quad P_{n}\left(x ; \Lambda_{n}\right)
$$

in $(0,1)$ strictly interlace. 
3. Numerical construction of the generalized Gaussian quadrature rule. Applying the theory of orthogonality for Müntz systems and using a method for evaluating orthogonal Müntz polynomials, in this section we present the method for the numerical construction of the generalized Gaussian quadrature rule for Müntz systems given by (1.7), i.e.,

$$
\int_{0}^{1} f(x) d \sigma(x)=\sum_{k=1}^{n} A_{k} f\left(x_{k}\right)+R_{n}(f)
$$

being exact for each $f \in M_{2 n-1}(\Lambda)$. The presented method is rather stable and performs calculations in double precision arithmetic to obtain double precision results.

Let $\Lambda=\left\{\lambda_{0}, \lambda_{1}, \lambda_{2}, \ldots\right\}$ be nondecreasing real sequence such that $\lambda_{\nu}>-1 / 2$ for every $\nu \in \mathbb{N}_{0}$. The orthogonal Müntz-Legendre system $\left\{P_{0}, P_{1}, \ldots, P_{2 n-1}\right\}$, defined by (2.2), is an EH system. It can be recognized as a linear span of the system of basis functions $x^{\Lambda}$, which is an EH system.

In what follows, by $P_{n}(x):=P_{n}(x ; \Lambda)$ we denote Müntz-Legendre polynomials given by (2.2), constructed for the nondecreasing real sequence $\Lambda=\left\{\lambda_{0}, \lambda_{1}, \ldots\right\}$. By $\sigma$ we denote a positive measure supported on the interval $[0,1]$. The use of orthogonal Müntz-Legendre polynomials in our procedure is very important. Our construction is quite different not only from the procedure for the classical Gaussian integration formulae with an algebraic degree of precision but also from the corresponding algorithm given in [13].

In the polynomial case the well-known Golub and Welsch procedure [9] is applied when the quadrature nodes $x_{k}$ are eigenvalues of the three-diagonal Jacobi matrix, i.e., the zeros of the (algebraic) polynomial $P_{n}(x)$ orthogonal with respect to the measure $d \sigma(x)$, and weights $A_{k}$ are expressed in terms of first component of the normalized eigenvectors corresponding to the eigenvalues $x_{k}$, respectively (cf. the routine GAUSS in the package ORTHPOL given by Gautschi [7]).

The algorithm in [13] for finding nodes and weights in (3.1) needs the construction of the functions $\xi_{i}$ and $\eta_{i}, i=1, \ldots, n$, defined by (1.3) and (1.4). The nodes $x_{1}, \ldots, x_{n}$ are Gaussian if and only if (see [13, Theorem 4.1])

$$
\int_{0}^{1} \xi_{i}(x) d \sigma(x)=\sum_{j=1}^{2 n} \alpha_{i j} \int_{0}^{1} P_{j-1}(x) d \sigma(x)=0, \quad i=1, \ldots, n .
$$

In this case, the Gaussian weights are given by

$$
A_{i}=\int_{0}^{1} \eta_{i}(x) d \sigma(x)=\sum_{j=1}^{2 n} \beta_{i j} \int_{0}^{1} P_{j-1}(x) d \sigma(x), \quad i=1, \ldots, n .
$$

Our algorithm can be summarized in the following steps.

We start with the initial system of nonlinear equations given by

$$
\sum_{k=1}^{n} A_{k} P_{\nu}\left(x_{k}\right)=\mu_{\nu}=\int_{0}^{1} P_{\nu}(x) d \sigma, \quad \nu=0,1, \ldots, 2 n-1 .
$$

Note that the vector of moments $\mu_{\nu}$ becomes quite simple, provided $d \sigma(x)=x^{\lambda_{0}} d x$. In this special case, according to the orthogonality condition of the Müntz-Legendre polynomials (see (2.6)), we have

$$
\mu_{\nu}=\frac{\delta_{\nu, 0}}{2 \lambda_{0}+1}, \quad \nu \in \mathbb{N}_{0}
$$


We rewrite the system of equations (3.2) in the form

$$
U_{n} \boldsymbol{A}-\boldsymbol{c}=\mathbf{0}, \quad V_{n} \boldsymbol{A}-\boldsymbol{d}=\mathbf{0},
$$

where $\boldsymbol{A}=\left[\begin{array}{llll}A_{1} & A_{2} & \cdots & A_{n}\end{array}\right]^{T}, \boldsymbol{c}=\left[\begin{array}{llll}\mu_{0} & \mu_{1} & \cdots & \mu_{n-1}\end{array}\right]^{T}$, and $\boldsymbol{d}=\left[\begin{array}{llll}\mu_{n} & \mu_{n+1} & \cdots & \mu_{2 n-1}\end{array}\right]^{T}$, and we apply the Newton-Kantorovich method in order to solve it.

Let $\Delta \boldsymbol{A}=\boldsymbol{A}-\hat{\boldsymbol{A}}$ and $\Delta \boldsymbol{x}=\boldsymbol{x}-\hat{\boldsymbol{x}}$, where $\hat{\boldsymbol{A}}$ and $\hat{\boldsymbol{x}}$ are the unique solutions of the starting nonlinear system. Then we can formulate the Newton-Kantorovich method as

$$
\begin{aligned}
& U_{n} \Delta \boldsymbol{A}+Y_{n} \Delta \boldsymbol{x}=U_{n} \boldsymbol{A}-\boldsymbol{c}, \\
& V_{n} \Delta \boldsymbol{A}+Z_{n} \Delta \boldsymbol{x}=V_{n} \boldsymbol{A}-\boldsymbol{d},
\end{aligned}
$$

or, equivalently,

$$
W_{n}\left[\begin{array}{c}
\Delta \boldsymbol{A} \\
\Delta \boldsymbol{x}
\end{array}\right]=\left[\begin{array}{c}
U_{n} \boldsymbol{A}-\boldsymbol{c} \\
V_{n} \boldsymbol{A}-\boldsymbol{d}
\end{array}\right],
$$

where

$$
\begin{aligned}
& W_{n}=\left[\begin{array}{ll}
U_{n} & Y_{n} \\
V_{n} & Z_{n}
\end{array}\right], \quad \boldsymbol{x}=\left[\begin{array}{llll}
x_{1} & x_{2} & \cdots & x_{n}
\end{array}\right]^{T}, \\
& U_{n}=U_{n}(\boldsymbol{x})=\left[\begin{array}{cccc}
P_{0}\left(x_{1}\right) & P_{0}\left(x_{2}\right) & \cdots & P_{0}\left(x_{n}\right) \\
P_{1}\left(x_{1}\right) & P_{1}\left(x_{2}\right) & & P_{1}\left(x_{n}\right) \\
\vdots & & & \\
P_{n-1}\left(x_{1}\right) & P_{n-1}\left(x_{2}\right) & & P_{n-1}\left(x_{n}\right)
\end{array}\right] \\
& V_{n}=V_{n}(\boldsymbol{x})=\left[\begin{array}{cccc}
P_{n}\left(x_{1}\right) & P_{n}\left(x_{2}\right) & \cdots & P_{n}\left(x_{n}\right) \\
P_{n+1}\left(x_{1}\right) & P_{n+1}\left(x_{2}\right) & & P_{n+1}\left(x_{n}\right) \\
\vdots & & & \\
P_{2 n-1}\left(x_{1}\right) & P_{2 n-1}\left(x_{2}\right) & & P_{2 n-1}\left(x_{n}\right)
\end{array}\right] \text {, } \\
& Y_{n}=Y_{n}(\boldsymbol{x})=\left[\begin{array}{cccc}
A_{1} P_{0}^{\prime}\left(x_{1}\right) & A_{2} P_{0}^{\prime}\left(x_{2}\right) & \cdots & A_{n} P_{0}^{\prime}\left(x_{n}\right) \\
A_{1} P_{1}^{\prime}\left(x_{1}\right) & A_{2} P_{1}^{\prime}\left(x_{2}\right) & & A_{n} P_{1}^{\prime}\left(x_{n}\right) \\
\vdots & & & \\
A_{1} P_{n-1}^{\prime}\left(x_{1}\right) & A_{2} P_{n-1}^{\prime}\left(x_{2}\right) & & A_{n} P_{n-1}^{\prime}\left(x_{n}\right)
\end{array}\right] \text {, } \\
& Z_{n}=Z_{n}(\boldsymbol{x})=\left[\begin{array}{cccc}
A_{1} P_{n}^{\prime}\left(x_{1}\right) & A_{2} P_{n}^{\prime}\left(x_{2}\right) & \cdots & A_{n} P_{n}^{\prime}\left(x_{n}\right) \\
A_{1} P_{n+1}^{\prime}\left(x_{1}\right) & A_{2} P_{n+1}^{\prime}\left(x_{2}\right) & & A_{n} P_{n+1}^{\prime}\left(x_{n}\right) \\
\vdots & & & \\
A_{1} P_{2 n-1}^{\prime}\left(x_{1}\right) & A_{2} P_{2 n-1}^{\prime}\left(x_{2}\right) & & A_{n} P_{2 n-1}^{\prime}\left(x_{n}\right)
\end{array}\right] .
\end{aligned}
$$

Now, our iterative process becomes

$$
\begin{aligned}
& \hat{\boldsymbol{x}}=\boldsymbol{x}-\left[V_{n} U_{n}^{-1} Y_{n}-Z_{n}\right]^{-1}\left(\boldsymbol{d}-V_{n} U_{n}^{-1} \boldsymbol{c}\right), \\
& \hat{\boldsymbol{A}}=U_{n}^{-1}\left[Y_{n}(\boldsymbol{x}-\hat{\boldsymbol{x}})+\boldsymbol{c}\right] .
\end{aligned}
$$


We can prove that our method for this construction is convergent. The basic theorem on the convergence of the Newton-Kantorovich method can be summarized as follows: Suppose we are given some differentiable function $\boldsymbol{f}: \mathbb{R}^{m} \mapsto \mathbb{R}^{m}, m \in \mathbb{N}$, with the property that at the solution of $\boldsymbol{f}(\boldsymbol{x})=0$, we have that $|J(\boldsymbol{x})| \neq 0$, i.e., the Jacobian of $\boldsymbol{f}$ at the solution is not singular. Then, there exists some $\varepsilon>0$, such that for any $\boldsymbol{y}_{0}$, with the property $\left\|\boldsymbol{y}_{0}-\boldsymbol{x}\right\|<\varepsilon$, we have that the sequence $\boldsymbol{y}_{k}, k \in \mathbb{N}$, defined by

$$
\boldsymbol{y}_{k+1}=\boldsymbol{y}_{k}-\left(J\left(\boldsymbol{y}_{k}\right)\right)^{-1} \boldsymbol{f}\left(\boldsymbol{y}_{k}\right), \quad k \in \mathbb{N}_{0},
$$

tends to $\boldsymbol{x}$ with a quadratic speed.

Lemma 3.1. Provided that the starting values are sufficiently good, the presented form of the Newton-Kantorovich method (3.5) is convergent. the form

Proof. The Jacobian for the starting nonlinear system can be easily calculated in

$$
J(\hat{\boldsymbol{x}}, \hat{\boldsymbol{A}})=W_{n} .
$$

It is easy to see that the determinant $\left|W_{n}\right|$ can be written as

$$
\left|W_{n}\right|=\left(\prod_{k=1}^{n} A_{k}\right)\left|\begin{array}{cccccc}
P_{0}\left(x_{1}\right) & \cdots & P_{0}\left(x_{n}\right) & P_{0}^{\prime}\left(x_{1}\right) & \cdots & P_{0}^{\prime}\left(x_{n}\right) \\
P_{1}\left(x_{1}\right) & & P_{1}\left(x_{n}\right) & P_{1}^{\prime}\left(x_{1}\right) & & P_{1}^{\prime}\left(x_{n}\right) \\
\vdots & & & & & \\
P_{2 n-1}\left(x_{1}\right) & & P_{2 n-1}\left(x_{n}\right) & P_{2 n-1}^{\prime}\left(x_{1}\right) & & P_{2 n-1}^{\prime}\left(x_{n}\right)
\end{array}\right| .
$$

Since our system of functions is $E H$ and the weights $A_{k}, k=1, \ldots, n$, are positive, this determinant cannot be zero, and thus the Jacobian has full rank. Also, since the system of functions $x^{\Lambda}$ is Chebyshev, the matrix $U_{n}$ is invertible. It is enough to apply the theorem on the matrix inversion in the block form presented in [18, p. 205] (see also [10, p. 13] and [26, p. 201]) to finish the proof.

The standard problem with the Newton-Kantorovich method is a problem of determining starting values. One way to ensure the convergence, for $d \sigma(x)=x^{\lambda_{0}} d x$, is based on the continuation of the classical Gaussian quadrature rule.

Lemma 3.2. Suppose we are given a nondecreasing system of real numbers $\Lambda=$ $\left\{\lambda_{k} \mid k \in \mathbb{N}_{0}\right\}, \lambda_{k}>-1 / 2, k \in \mathbb{N}_{0}$. The maps $A_{k}:=A_{k}(\alpha), x_{k}:=x_{k}(\alpha), \alpha \in[0,1]$, defined by the nonlinear system of equations

$$
\sum_{k=1}^{n} A_{k} P_{\nu}^{\alpha}\left(x_{k}\right)=\frac{\delta_{\nu, 0}}{2 \alpha \lambda_{0}+1}, \quad \nu=0,1, \ldots, 2 n-1
$$

where

$$
P_{\nu}^{\alpha}(x):=P_{\nu}\left(x ;(1-\alpha) \mathbb{N}_{0}+\alpha \Lambda\right)=\frac{1}{2 \pi i} \oint_{\Gamma} G_{\nu}(s, \alpha, \Lambda) x^{s} d s
$$

and

$$
G_{\nu}(s, \alpha, \Lambda)=\prod_{k=0}^{\nu-1} \frac{s+(1-\alpha) k+\alpha \lambda_{k}+1}{s-(1-\alpha) k-\alpha \lambda_{k}} \frac{1}{s-(1-\alpha) \nu-\alpha \lambda_{\nu}},
$$

are continuous. 
Proof. It is easy to verify that the function

$$
\boldsymbol{F}(\boldsymbol{x}, \boldsymbol{A}, \alpha)=\left[\begin{array}{c}
\sum_{k=1}^{n} A_{k} P_{0}^{\alpha}\left(x_{k}\right) \\
\vdots \\
\sum_{k=1}^{n} A_{k} P_{2 n-1}^{\alpha}\left(x_{k}\right)
\end{array}\right]
$$

has derivatives in $x_{k}, A_{k}, k=1, \ldots, n$, and $\alpha$ for $0<x_{1}<x_{2}<\cdots<x_{n}<1$, $A_{k}>0, k=1, \ldots, n$, and $\alpha \in(0,1)$. We have

$$
\begin{aligned}
\partial_{x_{j}}\left(\sum_{k=1}^{n} A_{k} P_{\nu}^{\alpha}\left(x_{k}\right)\right) & =A_{j}\left(P_{\nu}^{\alpha}\right)^{\prime}\left(x_{j}\right), \quad \nu=0,1, \ldots, 2 n-1, \quad j=1, \ldots, n, \\
\partial_{A_{j}}\left(\sum_{k=1}^{n} A_{k} P_{\nu}^{\alpha}\left(x_{k}\right)\right) & =P_{\nu}^{\alpha}\left(x_{j}\right), \quad \nu=0,1, \ldots, 2 n-1, \quad j=1, \ldots, n, \\
\partial_{\alpha}\left(\sum_{k=1}^{n} A_{k} P_{\nu}^{\alpha}\left(x_{k}\right)\right) & =\frac{1}{2 \pi i} \oint_{\Gamma} \partial_{\alpha}\left\{\left(G_{\nu}(s, \alpha, \Lambda)\right)\left(\sum_{k=1}^{n} A_{k} x_{k}^{s}\right)\right\} d s .
\end{aligned}
$$

Since for every $\alpha \in[0,1]$, there exists the unique solution of

$$
\boldsymbol{F}(\boldsymbol{A}, \boldsymbol{x}, \alpha)=\frac{\boldsymbol{e}_{0}}{2 \alpha \lambda_{0}+1}
$$

in $\boldsymbol{A}$ and $\boldsymbol{x}$, we can define the functions $A_{k}:=A_{k}(\alpha), x_{k}:=x_{k}(\alpha), k=1, \ldots, n$. Here, $\boldsymbol{e}_{0}$ is the fist coordinate vector, i.e., $\boldsymbol{e}_{0}=\left[\begin{array}{llll}1 & 0 & \cdots & 0\end{array}\right]^{T}$.

Using the system of equations (3.6), we can calculate derivatives with respect to $\alpha$ in the form

$$
\partial_{\alpha} \boldsymbol{F}+\sum_{k=1}^{n}\left(\partial_{x_{k}} \boldsymbol{F} \frac{d x_{k}}{d \alpha}+\partial_{A_{k}} \boldsymbol{F} \frac{d A_{k}}{d \alpha}\right)=-\frac{2 \lambda_{0}}{\left(2 \alpha \lambda_{0}+1\right)^{2}} \boldsymbol{e}_{0} .
$$

Now, from here we get

$$
W_{n}\left[\begin{array}{c}
\frac{d A_{1}}{d \alpha} \\
\vdots \\
\frac{d x_{n}}{d \alpha}
\end{array}\right]=-\frac{2 \lambda_{0}}{\left(2 \alpha \lambda_{0}+1\right)^{2}} \boldsymbol{e}_{0}-\partial_{\alpha} \boldsymbol{F}
$$

where the matrix $W_{n}$ is the same as in (3.4), formed with $P_{\nu}$ replaced by $P_{\nu}^{\alpha}$. Since the matrix $W_{n}$ is invertible, it is easy to conclude that the functions $A_{k}:=A_{k}(\alpha)$, $x_{k}:=x_{k}(\alpha), k=1, \ldots, n$, are differentiable.

Since the functions $A_{k}(\alpha)$ and $x_{k}(\alpha), k=1, \ldots, n$, are continuous in $\alpha \in[0,1]$, we start with $\alpha=0$ and construct the classical Gaussian quadrature rule with parameters $A_{k}(0)$ and $x_{k}(0)$. Now, if we increase $\alpha$ by a small amount, we can use the obtained values for $A_{k}(0)$ and $x_{k}(0)$ as starting values for $A_{k}(\alpha)$ and $x_{k}(\alpha)$ in the NewtonKantorovich method. Again, we can increase $\alpha$ by a small amount and try to solve the system with starting values obtained from the previous step. We repeat the procedure until $\alpha=1$ is reached. 
There is still another possibility of continuation. Namely, we have the following lemma.

Lemma 3.3. Let $\boldsymbol{\mu}=\left[\begin{array}{llll}\mu_{0} & \mu_{1} & \cdots & \mu_{2 n-1}\end{array}\right]^{T}$ be a vector of moments defined by (3.2). The functions $A_{k}:=A_{k}(\boldsymbol{\mu})$ and $x_{k}:=x_{k}(\boldsymbol{\mu}), k=1, \ldots, n$, given by the nonlinear system of equations (3.2) are continuous.

Proof. Note that by the fact that there exists a unique solution to the system of equations (3.2), we have that $A_{k}$ and $x_{k}$ are single valued functions. Since the Jacobi matrix $W_{n}$ is invertible at any solution, according to the inverse function theorem, we have that $x_{k}$ and $A_{k}, k=1, \ldots, n$, are continuous in $\boldsymbol{\mu}$.

Using this lemma and the following idea we can construct the generalized Gaussian quadrature rule for some measure $\sigma$. Denote moments for the measure $x^{\lambda_{0}} d x$ by $\delta_{\nu, 0} /\left(2 \lambda_{0}+1\right), \nu=0,1, \ldots, 2 n-1$. By $\boldsymbol{\mu}$ integrals as in (3.2), we can introduce the moments

$$
\hat{\mu}_{\nu}=(1-\alpha) \frac{\delta_{\nu, 0}}{2 \lambda_{0}+1}+\alpha \mu_{\nu}, \quad \nu=0,1, \ldots, 2 n-1, \quad \alpha \in[0,1] .
$$

Then $x_{k}$ and $A_{k}, k=1, \ldots, n$, are continuous functions of $\alpha \in[0,1]$. Thus, we increase $\alpha$ by a small amount and solve the systems of nonlinear equations (3.2) in order to achieve $\alpha=1$ starting with $\alpha=0$.

According to the previous two lemmas, we can construct the solution using the following steps:

- First, using Lemma 3.2, starting from the classical Gauss-Legendre quadrature rule, we construct the generalized quadrature rule for the $x^{\lambda_{0}} d x$ measure and any given system $\Lambda$.

- Once the generalized quadrature rule is constructed for the $x^{\lambda_{0}} d x$ measure, we introduce the moments, as given in (3.7), to construct the generalized quadrature rule for the targeting measure $\sigma$, with the moment vector $\boldsymbol{\mu}$.

We next address numerical properties of the nonlinear system of equations (3.2). For example, we have to give the algorithm to calculate the values of the polynomials $P_{\nu}, \nu=0,1, \ldots, 2 n-1$, at the points $x_{k}, k=1, \ldots, n$. This is already solved (see [17]), and we address it in subsection 3.3. For derivatives $P_{k}^{\prime}(x)$ we can use the recurrence relation (2.8), i.e.,

$$
x P_{n}^{\prime}(x)-x P_{n-1}^{\prime}(x)=\lambda_{n} P_{n}(x)+\left(1+\bar{\lambda}_{n-1}\right) P_{n-1}(x) .
$$

It is easy to verify that our method depends strongly on the condition number of the matrix $U_{n}$. In fact, assuming the Newton-Kantorovich method is converging, it is easy to verify that the weights $\boldsymbol{A}$ are the solution of the system of linear equations

$$
U_{n} \boldsymbol{A}=\frac{\boldsymbol{e}_{0}}{2 \lambda_{0}+1},
$$

where the matrix $U_{n}=\left[P_{\nu}\left(x_{k}\right)\right]_{\nu, k=1}^{n}$ is calculated at the solution for the nodes $x_{k}$, $k=1, \ldots, n$. Hence, the precision in constructed weights $A_{k}, k=1, \ldots, n$, can be determined according to the condition number of the matrix $U_{n}$. Note that the precision in the constructed nodes $x_{k}, k=1, \ldots, n$, is also determined by the precision in the constructed weights $A_{k}$. To present this simple fact, we consider our system of equations for the Newton-Kantorovich method (3.5). We have

$$
\begin{aligned}
\hat{x} & =x-\left(V_{n} U_{n}^{-1} Y_{n}-Z_{n}\right)^{-1}\left(\boldsymbol{d}-V_{n} U_{n}^{-1} \boldsymbol{c}\right) \\
& =x-\left(V_{n} U_{n}^{-1} Y_{n}-Z_{n}\right)^{-1}\left(\boldsymbol{d}-V_{n} \overline{\boldsymbol{A}}\right)=x-\left(V_{n} U_{n}^{-1} Y_{n}-Z_{n}\right)^{-1}(\boldsymbol{d}-\overline{\boldsymbol{d}}),
\end{aligned}
$$


where

$$
\overline{\boldsymbol{d}}=V_{n} \overline{\boldsymbol{A}}
$$

is a vector of the calculated moments $\left[\bar{\mu}_{n} \cdots \bar{\mu}_{2 n-1}\right]^{T}$ with weights $\overline{\boldsymbol{A}}$. Obviously we have $\boldsymbol{d} \neq \overline{\boldsymbol{d}}$, which has as a consequence that nodes $\boldsymbol{x}$ are constructed with an error originating from the fact that weights cannot be constructed without an error.

3.1. Condition number of $\boldsymbol{U}_{\boldsymbol{n}}$. The algorithm strongly depends on the condition number of the matrix $U_{n}$. Assuming all nodes $\boldsymbol{x}$ are known, it is obvious that we can calculate the weights $\boldsymbol{A}$ as $U_{n}^{-1} \boldsymbol{c}$. Although it is hard to give precise results about the condition number of $U_{n}$, for some special cases linked with polynomials, we can give some theoretical results concerning the condition number of this matrix.

We define the condition number of a matrix $B$ to be $\|B\| \mid\left\|B^{-1}\right\|$, where, as it is known, any matrix norm will do. Following [8, p. 64], we restrict our attention to the Frobenius norm, i.e., $\left\|\left[b_{i, j}\right]_{i, j=1}^{n}\right\|=\left(\sum_{i} \sum_{j} b_{i, j}^{2}\right)^{1 / 2}$. We consider the slightly modified matrix $U_{n}$, i.e., we put the normalized version of Müntz-Legendre polynomials into the system of nonlinear equations (3.2) so that we have

$$
\sum_{k=1}^{n} A_{k} p_{\nu}\left(x_{k}\right)=\frac{\mu_{\nu}}{\left\|P_{\nu}\right\|}=\int_{0}^{1} p_{\nu}(x) d \sigma(x), \quad \nu=0,1, \ldots, 2 n-1 .
$$

This has as a consequence that in matrices $U_{n}, V_{n}, Y_{n}$, and $Z_{n}, P_{\nu}$ is replaced with $p_{\nu}, \nu=0,1, \ldots, 2 n-1$, and vectors $\boldsymbol{d}$ and $\boldsymbol{c}$ are filled with the sequence of moments $\mu_{\nu} /\left\|P_{\nu}\right\|, \nu \in \mathbb{N}_{0}$. This renormalization of the system does not change anything, as is explained in [8, pp. 65-66].

In this case, we can express

$$
\left\|U_{n}\right\|^{2}=\sum_{k=1}^{n} \sum_{\nu=0}^{n-1} p_{\nu}\left(x_{k}\right)^{2} .
$$

In order to get the inverse matrix, we define Müntz-Lagrange polynomials as

$$
\ell_{k}(x)=\sum_{\nu=0}^{n-1} \alpha_{k}^{\nu} p_{\nu}(x), \quad k=1, \ldots, n,
$$

with the property

$$
\ell_{k}\left(x_{\nu}\right)=\delta_{k, \nu}, \quad k, \nu=1, \ldots, n .
$$

The Müntz-Lagrange polynomials $\ell_{k}, k=1, \ldots, n$, are defined correctly since $p_{\nu}, \nu=$ $0,1, \ldots, n-1$, makes a Chebyshev system. Also, it is easy to prove that $\operatorname{span}\left\{\ell_{k} \mid k=\right.$ $1, \ldots, n\}$ is exactly the same as $\operatorname{span}\left\{p_{\nu} \mid \nu=0,1, \ldots, n-1\right\}$.

Now, we can identify the matrix $U_{n}^{-1}$ as the matrix $\left[\alpha_{k}^{\nu}\right]_{\nu=0,1, \ldots, n-1, k=1, \ldots, n}$, and then we can calculate the norm of the matrix $U_{n}^{-1}$ in the form

$$
\left\|U_{n}^{-1}\right\|^{2}=\sum_{\nu=0}^{n-1} \sum_{k=1}^{n}\left(\alpha_{k}^{\nu}\right)^{2}
$$

Using the simple identity

$$
\int_{0}^{1} \ell_{k}(x)^{2} d x=\int_{0}^{1}\left\{\sum_{\nu=0}^{n-1} \alpha_{k}^{\nu} p_{\nu}(x) \sum_{\mu=0}^{n-1} \alpha_{k}^{\mu} p_{\mu}(x)\right\} d x=\sum_{\nu=0}^{n-1}\left(\alpha_{k}^{\nu}\right)^{2},
$$


we get

$$
\left\|U_{n}^{-1}\right\|^{2}=\int_{0}^{1}\left(\sum_{k=1}^{n} \ell_{k}(x)^{2}\right) d x .
$$

In total we express the condition number of the matrix $U_{n}$ as

$$
\left\|U_{n}\right\|\left\|U_{n}^{-1}\right\|=\left(\sum_{k=1}^{n} \sum_{\nu=0}^{n-1} p_{\nu}\left(x_{k}\right)^{2}\right)^{1 / 2}\left(\int_{0}^{1}\left(\sum_{k=1}^{n} \ell_{k}(x)^{2}\right) d x\right)^{1 / 2} .
$$

It is not easy to give some estimates of this number; hence, we are going to discuss two cases connected to the orthogonal polynomials. The first is for $\Lambda=\left\{k \mid k \in \mathbb{N}_{0}\right\}$, where our Müntz-Legendre polynomials are really Legendre polynomials. The second case is for $\Lambda=\left\{0 \mid k \in \mathbb{N}_{0}\right\}$, where our Müntz-Legendre polynomials are Laguerre polynomials in $-\log x$, as given in (2.5). As experiments show, the behavior for the general nondecreasing sequence $\Lambda$ can be understood using these two special cases. Hence, we discuss these two cases.

According to the fact that in these cases Müntz-Lagrange polynomials can be expressed in the form

$$
\ell_{\nu}(x)=\frac{p_{n}(x)}{\left(x-x_{\nu}\right) p_{n}^{\prime}\left(x_{\nu}\right)}, \quad \nu=1, \ldots, n,
$$

for the Legendre case, and as

$$
\ell_{\nu}(x)=\frac{p_{n}(x)}{\left(-\log x-x_{\nu}\right) p_{n}^{\prime}\left(x_{\nu}\right)}, \quad \nu=1, \ldots, n,
$$

in the Laguerre case, we have an orthogonality of the Müntz-Lagrange polynomials, i.e.,

$$
\int_{0}^{1} \ell_{\nu}(x) \ell_{k}(x) d x=\left\|\ell_{k}\right\|^{2} \delta_{\nu, k}, \quad \nu, k=1, \ldots, n .
$$

Then, according to the fact that $1=\sum_{\nu} \ell_{\nu}(x)$, and using the mentioned orthogonality, we find

$$
\int_{0}^{1}\left(\sum_{\nu=1}^{n} \ell_{\nu}(x)^{2}\right) d x=\int_{0}^{1}\left(\sum_{\nu=1}^{n} \ell_{\nu}(x) \sum_{k=1}^{n} \ell_{k}(x)\right) d x=\int_{0}^{1}\left(\sum_{\nu=1}^{n} \ell_{\nu}(x)\right)^{2} d x=1,
$$

so that $\left\|U_{n}^{-1}\right\|=1$.

Using the Shohat formula (see [19], [23]), we can express the norm $\left\|U_{n}\right\|$ in the form

$$
\left\|U_{n}\right\|^{2}=\sum_{k=1}^{n} \frac{1}{w_{k}}
$$

where we denoted by $w_{k}, k=1, \ldots, n$, the weights in the classical Gaussian quadrature rule associated with the sequence of polynomials $p_{\nu}, \nu \in \mathbb{N}_{0}$. Using the Markov inequality (see $[24$, p. 50])

$$
\sum_{k=\nu+1}^{n} w_{k} \leq \int_{x_{\nu}}^{+\infty} d \mu(x), \quad \nu=1, \ldots, n-1
$$


we have, for the Laguerre measure, $\exp \left(x_{n-1}\right) \leq\left(w_{n}\right)^{-1}$, which means that in the Laguerre case we have $\left\|U_{n}\right\| \geq \exp (\sqrt{n} / 2)$, where we have used an estimate for zeros of Laguerre polynomials given in [24, p. 129]. Hence, for the sequence $\Lambda=\left\{0 \mid k \in \mathbb{N}_{0}\right\}$, the condition number of the matrix $U_{n}$ grows exponentially with $n$.

In what follows, for two sequences $A_{n}$ and $B_{n}$ we write $A_{n} \sim B_{n}$ if and only if there exist two positive constants $C_{1}$ and $C_{2}$ such that $C_{1} \leq\left|A_{n} / B_{n}\right| \leq C_{2}$.

Now, for the Legendre measure we use the following asymptotic estimate $w_{k} \sim$ $\pi\left(1-x_{k}^{2}\right)^{1 / 2} / n, k=1, \ldots, n$, given in [21, p. 85] (see also Szegö [24, p. 253]), to get

$$
\left\|U_{n}\right\|^{2}=\sum_{k=1}^{n} \frac{1}{w_{k}} \sim \frac{n}{\pi} \sum_{k=1}^{n}\left(1-x_{k}^{2}\right)^{-1 / 2}
$$

Using the estimate for the zeros $x_{k}=-\cos \theta_{k}, k=1, \ldots, n$, of the Legendre polynomials (see $[24$, p. 125])

$$
\frac{k-1 / 2}{n+1 / 2}<\theta_{k}<\frac{k}{n+1 / 2}, \quad k=1, \ldots, n,
$$

and Jordan inequality $2 \theta / \pi<\sin \theta<\theta, \theta \in(0, \pi / 2)$, we have simply

$$
\left\|U_{n}\right\|^{2} \sim \frac{n}{\pi} \sum_{k=1}^{n} \frac{1}{\sin \theta_{k}} \sim \frac{2 n}{\pi} \sum_{k=1}^{[n / 2]} \frac{1}{\sin \theta_{k}} \sim n \sum_{k=1}^{[n / 2]} \frac{1}{\theta_{k}} \sim n\left(n+\frac{1}{2}\right) \sum_{k=1}^{[n / 2]} \frac{1}{k} \sim n^{2} \log n,
$$

so that, for the Legendre case, i.e., $\Lambda=\left\{k \mid k \in \mathbb{N}_{0}\right\}$, we have that the condition number of the matrix $U_{n}$ grows like $n \log ^{1 / 2} n$.

LEMmA 3.4. If $\Lambda=\left\{k \mid k \in \mathbb{N}_{0}\right\}$, we have $\left\|U_{n}\right\|\left\|U_{n}^{-1}\right\| \sim n \log ^{1 / 2} n$. If $\Lambda=$ $\left\{0 \mid k \in \mathbb{N}_{0}\right\}$, we have ||$U_{n}|||| U_{n}^{-1}|| \sim \exp (\sqrt{n} / 2)$.

This lemma shows that in the cases when a nondecreasing sequence $\Lambda$ is close to the Legendre case, we should expect the condition number of $U_{n}$ to grow relatively slowly with respect to $n$. In the opposite case for the almost constant sequence $\Lambda$, we should expect the condition number to grow rather rapidly with respect to $n$. This behavior is going to be illustrated in the last section.

3.2. Construction for the nonintegrable basis. In what follows we discuss the construction of the generalized Gaussian quadrature rule in the case when a nondecreasing sequence $\Lambda$ is such that for some $\lambda_{k}$ we have $2 \lambda_{k} \leq-1$. It is easy to verify that in this case we cannot construct the sequence of Müntz-Legendre polynomials orthogonal with respect to the Legendre measure since the functions $x^{\lambda_{k}} x^{\lambda_{k}}$, for which $2 \lambda_{k} \leq-1$, are not integrable with respect to the Legendre measure.

What we can do is use Müntz polynomials given by (2.7), which are orthogonal with respect to the measure $x^{\beta} d x$, where $\beta$ is chosen such that we have $2 \lambda_{k}+\beta>-1$, $k \in \mathbb{N}_{0}$. If we introduce a new nondecreasing sequence $\Lambda+\beta / 2$, we can construct the sequence of Müntz polynomials $P_{n}(\cdot ; \Lambda+\beta / 2), n \in \mathbb{N}_{0}$, generated by $\Lambda+\beta / 2$, orthogonal with respect to Legendre measure, and then, using the previously described method, we are able to solve the system of equations

$$
\sum_{k=1}^{n} A_{k} P_{\nu}\left(x_{k} ; \Lambda+\frac{\beta}{2}\right)=\frac{\delta_{\nu, 0}}{2 \lambda_{0}+\beta+1}=\int_{0}^{1} x^{\lambda_{0}+\beta / 2} P_{\nu}\left(x ; \Lambda+\frac{\beta}{2}\right) d x, 0 \leq \nu \leq 2 n-1 .
$$


Then, if we rewrite the system in the form

$$
\begin{aligned}
& \sum_{k=1}^{n}\left(A_{k} x_{k}^{\beta / 2}\right)\left(x_{k}^{-\beta / 2} P_{\nu}\left(x_{k} ; \Lambda+\frac{\beta}{2}\right)\right) \\
& \quad=\frac{1}{2 \lambda_{0}+\beta+1} \delta_{\nu, 0}=\int_{0}^{1} x^{\lambda_{0}+\beta}\left(x^{-\beta / 2} P_{\nu}\left(x ; \Lambda+\frac{\beta}{2}\right)\right) d x, \quad \nu=0,1, \ldots, 2 n-1
\end{aligned}
$$

and if we choose

$$
A_{k}^{*}=A_{k} x_{k}^{\beta / 2}, \quad x_{k}^{*}=x_{k}, \quad k=1, \ldots, n,
$$

it can be verified that the quadrature rule

$$
\sum_{k=1}^{n} A_{k}^{*} f\left(x_{k}^{*}\right)
$$

is exact in the linear span of $x^{\Lambda_{2 n-1}}$ with respect to the measure $x^{\lambda_{0}+\beta} d x$. Thus, we have the following result.

Lemma 3.5. Let a nondecreasing sequence $\Lambda+\beta / 2, \beta \in \mathbb{R}$, of the real numbers be given and let $x_{k}$ and $A_{k}, k=1, \ldots, n$, be nodes and weights in the generalized quadrature rule (3.2) with respect to the measure $x^{\lambda_{0}+\beta / 2} d x$. The generalized Gaussian quadrature rule (3.2) for the measure $x^{\lambda_{0}+\beta} d x$, which is exact in $x^{\Lambda_{2 n-1}}$, has nodes and weights given by

$$
x_{k}^{*}=x_{k}, \quad A_{k}^{*}=A_{k} x_{k}^{\beta / 2}, \quad k=1, \ldots, n .
$$

A consequence of this simple observation is the possibility of using the described algorithm for the construction of the generalized Gaussian quadrature rule, for example, for the set of functions

$$
x^{k-2 / 3}, \quad x^{k}, \quad k=0,1, \ldots, 2 n-1 .
$$

Some numerical values for this case are presented in the last section.

3.3. Numerical computation of Müntz-Legendre polynomials. A direct evaluation of Müntz polynomials $P_{n}(x)$ in the power form (2.3) can be a big problem in finite arithmetic, especially when $n$ is a large number and $x$ is close to 1 . These problems have been addressed in [17].

In this subsection we give a stable numerical method for evaluating the values of the Müntz-Legendre polynomials defined by (2.1) and (2.2), i.e.,

$$
P_{n}(x)=\frac{1}{2 \pi i} \oint_{\Gamma} W_{n}(s) x^{s} d s, \quad W_{n}(s)=\prod_{\nu=0}^{n-1} \frac{s+\bar{\lambda}_{\nu}+1}{s-\lambda_{\nu}} \cdot \frac{1}{s-\lambda_{n}} .
$$

For a detailed discussion, see [17].

Our method is based on a direct evaluation of the contour integral in (3.8). At first we take the contour $\Gamma=\Gamma_{R}=C_{R} \cup L_{R}$ (see Figure 3.1 (left)). As we can see, $C_{R}$ is a semicircle with the center at $\sigma<-1 / 2$, its radius $R$ is such that all poles of $W_{n}(s)$ are inside the contour $\Gamma_{R}$, and $L_{R}$ is a part of the straight line $s=\sigma+i t$, $-R \leq t \leq R$. (Notice that the function $W_{n}(s)$ in Figure 3.1 has only real poles.) 

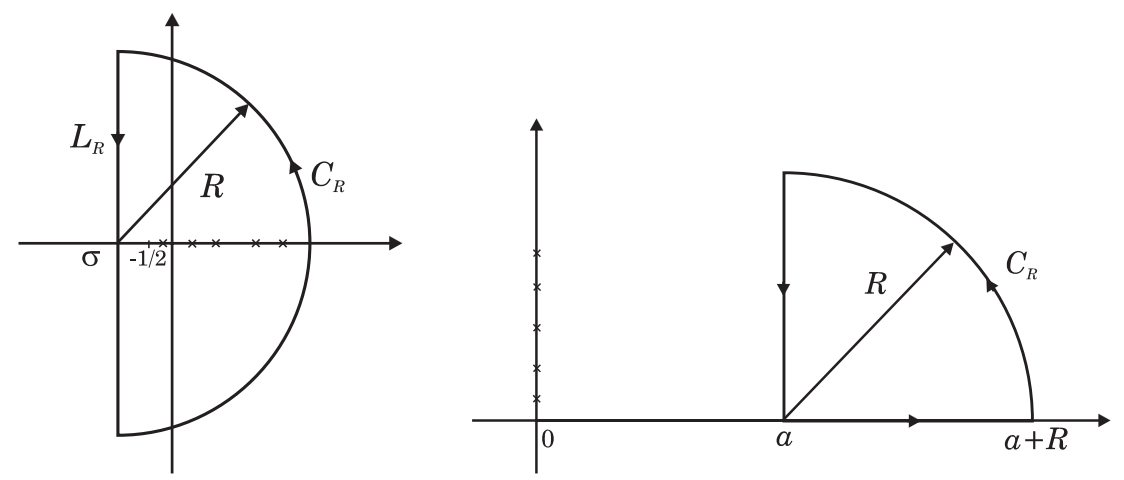

FIG. 3.1. The contour of integration for the integral in (3.1) (left) and for the integral $L_{2}\left(f_{n}(\cdot ; \omega)\right)($ right $)$.

We have a simple lemma.

LEMMA 3.6. $\int_{C_{R}} W_{n}(s) x^{s} d s \rightarrow 0$ when $R \rightarrow+\infty$.

Thus, when $R \rightarrow+\infty$, an integration along the contour $\Gamma_{R}$ reduces to the integration over the line $L_{R}$, so that

$$
P_{n}(x)=\frac{x^{\sigma}}{2 \pi i} \int_{-\infty}^{+\infty} f_{n}(t ; \omega) e^{i t} d t,
$$

where $\omega=\log (1 / x)>0$ and

$$
f_{n}(t ; \omega)=\prod_{\nu=0}^{n-1} \frac{t+i\left(\sigma+\bar{\lambda}_{\nu}+1\right) \omega}{t+i\left(\sigma-\lambda_{\nu}\right) \omega} \cdot \frac{1}{t+i\left(\sigma-\lambda_{n}\right) \omega} .
$$

This gives the following result.

Theorem 3.7. Let $\sigma<-1 / 2, \omega=\log (1 / x), f_{n}(t ; \omega)$ be defined by (3.9), and

$$
\varphi_{n}(t ; \omega)=\frac{1}{2 i}\left(f_{n}(t ; \omega) e^{i t}+f_{n}(-t ; \omega) e^{-i t}\right) .
$$

The Müntz-Legendre polynomials can be represented in the one-side integral form

$$
P_{n}(x)=\frac{x^{\sigma}}{\pi} \int_{0}^{+\infty} \varphi_{n}(t ; \omega) d t .
$$

In what follows we consider the case when the sequence $\Lambda$ is real. An important corollary of Theorem 3.7 is the following result.

THEOREM 3.8. Let $\Lambda=\left\{\lambda_{0}, \lambda_{1}, \lambda_{2}, \ldots\right\}$ be a real sequence such that $\lambda_{\nu}>-1 / 2$ for every $k \in \mathbb{N}_{0}, f_{n}(t ; \omega)$ is defined by $(3.9), \omega=\log (1 / x)$, and $\sigma<-1 / 2$. Then the Müntz-Legendre polynomials have the integral representation

$$
P_{n}(x)=\frac{x^{\sigma}}{\pi} \operatorname{Im}\left\{\int_{0}^{+\infty} f_{n}(t ; \omega) e^{i t} d t\right\} .
$$

In this case we have $f_{n}(-t ; \omega)=\overline{f_{n}(t ; \omega)}$, then $(3.10)$ becomes $\varphi_{n}(t ; \omega)=$ $\operatorname{Im}\left\{f_{n}(t ; \omega) e^{i t}\right\}$, and (3.11) gives (3.12). The poles of $f_{n}(t ; \omega)$ are then purely imaginary, $i\left(\lambda_{\nu}-\sigma\right) \omega, \nu=0,1, \ldots, n$, and located on the positive part of the imaginary axes, because of $\lambda_{\nu}>\sigma$ and $\omega=\log (1 / x)>0$. 
In order to calculate the integral in (3.12) (or in (3.11)) we use an idea from complex integration (see [16, Thm. 2.2]). We select a positive number $a=m \pi(m \in \mathbb{N})$ and put

$$
\begin{aligned}
\int_{0}^{+\infty} f_{n}(t ; \omega) e^{i t} d t & =\int_{0}^{a} f_{n}(t ; \omega) e^{i t} d t+\int_{a}^{+\infty} f_{n}(t ; \omega) e^{i t} d t \\
& =L_{1}\left(f_{n}(\cdot ; \omega)\right)+L_{2}\left(f_{n}(\cdot ; \omega)\right) .
\end{aligned}
$$

Here,

$$
L_{1}\left(f_{n}(\cdot ; \omega)\right)=\int_{0}^{a=m \pi} f_{n}(t ; \omega) e^{i t} d t=\sum_{k=1}^{m} \int_{(k-1) \pi}^{k \pi} f_{n}(t ; \omega) e^{i t} d t
$$

i.e.,

$$
L_{1}\left(f_{n}(\cdot ; \omega)\right)=\pi \int_{0}^{1}\left[\sum_{k=1}^{m} f_{n}(\pi(\xi+k-1) ; \omega) e^{i \pi(\xi+k-1)}\right] d \xi,
$$

and $L_{2}\left(f_{n}(\cdot ; \omega)\right)=\int_{a}^{+\infty} f_{n}(t ; \omega) e^{i t} d t$.

Since $f_{n}(z ; \omega)$ is a holomorphic function in the region $D=\{z \in \mathbb{C} \mid \operatorname{Re} z \geq a>$ $0, \operatorname{Im} z \geq 0\}$ and $\left|f_{n}(z ; \omega)\right| \leq A /|z|$ when $|z| \rightarrow+\infty$, for some positive constant $A$, we can prove

$$
L_{2}\left(f_{n}(\cdot ; \omega)\right)=i e^{i a} \int_{0}^{+\infty} f_{n}(a+i y ; \omega) e^{-y} d y
$$

For $a=m \pi,(3.14)$ becomes

$$
L_{2}\left(f_{n}(\cdot ; \omega)\right)=(-1)^{m} \int_{0}^{+\infty} \psi_{n}(y ; \omega) e^{-y} d y
$$

where

$$
\psi_{n}(y ; \omega)=i f_{n}(a+i y ; \omega)=\prod_{\nu=0}^{n-1} \frac{y+\left(\sigma+\lambda_{\nu}+1\right) \omega-i a}{y+\left(\sigma-\lambda_{\nu}\right) \omega-i a} \cdot \frac{1}{y+\left(\sigma-\lambda_{n}\right) \omega-i a} .
$$

TheOREM 3.9. Under conditions from Theorem 3.8, the Müntz-Legendre polynomials have a computable representation

$$
P_{n}(x)=\frac{x^{\sigma}}{\pi} \operatorname{Im}\left\{L_{1}\left(f_{n}(\cdot ; \omega)\right)+L_{2}\left(f_{n}(\cdot ; \omega)\right)\right\},
$$

where $L_{1}\left(f_{n}(\cdot ; \omega)\right)$ and $L_{2}\left(f_{n}(\cdot ; \omega)\right)$ are given by $(3.13)$ and $(3.15)$, respectively.

In the numerical implementation we use the Gauss-Legendre rule on $(0,1)$ and the Gauss-Laguerre rule for calculating $L_{1}\left(f_{n}(\cdot ; \omega)\right)$ and $L_{2}\left(f_{n}(\cdot ; \omega)\right)$, respectively. A convenient choice for the parameter $\sigma$ is $\lambda_{\min }-\pi / \omega$, where $\lambda_{\min }=\min \left\{\lambda_{0}, \lambda_{1}, \ldots\right\}$.

4. Numerical examples. In this section we present some numerical examples. Here we want to emphasize that in all examples, as starting values for the NewtonKantorovich method (3.2), we used the zeros of the Müntz-Legendre polynomial $P_{n}$. According to Theorem 2.4 there are exactly $n$ different zeros of $P_{n}$ in the interval $(0,1)$. 
TABLE 4.1

Nodes and weights in the generalized Gaussian quadrature rule for $\lambda_{2 k}=k$ and $\lambda_{2 k+1}=k+1 / 3$, $k \in \mathbb{N}_{0}$ (left) and $\lambda_{2 k}=\lambda_{2 k+1}=k, k \in \mathbb{N}_{0}$ (right) for 30 points.

\begin{tabular}{|c|c|c|c|}
\hline Nodes & Weights & Nodes & Weights \\
\hline $.11890626221894(-4)$ & $.41297515794865(-4)$ & $.73237974427261(-5)$ & $.27989215430955(-4)$ \\
\hline $.14403587843195(-3)$ & $.26627367672116(-3)$ & $.11004470045777(-3)$ & $.21736552650254(-3)$ \\
\hline $.65503119332169(-3)$ & $.82242421680232(-3)$ & $.54691832618397(-3)$ & $.72070358653439(-3)$ \\
\hline $.19430375893262(-2)$ & $.18402261193412(-2)$ & $.17018575191016(-2)$ & $.16744609650550(-2)$ \\
\hline $.45251469339122(-2)$ & $.34260127408358(-2)$ & $.40838636097144(-2)$ & $.31912824064115(-2)$ \\
\hline $.90102163603464(-2)$ & $.56560992521664(-2)$ & $.83000411768823(-2)$ & $.53537883135293(-2)$ \\
\hline $.16066432118835(-1)$ & $.85721821462782(-2)$ & $.15022978156080(-1)$ & $.82096213680820(-2)$ \\
\hline $.26385011600158(-1)$ & $.12178245807910(-1)$ & $.24953923615755(-1)$ & $.11768029213085(-1)$ \\
\hline $.40641627763273(-1)$ & $.16439118767071(-1)$ & $.38783386171063(-1)$ & $.15998143504891(-1)$ \\
\hline $.59457247937461(-1)$ & $.21280752356760(-1)$ & $.57150898481176(-1)$ & $.20829041093624(-1)$ \\
\hline $.83360115784155(-1)$ & $.26592222426836(-1)$ & $.80605741472655(-1)$ & $.26151597661309(-1)$ \\
\hline $.11275056965256(-1)$ & $9382586004(-1)$ & .10 & $.31822068269456(-1)$ \\
\hline .14787028400 & $.38020028095164(-1)$ & .1443083730 & $.37667255999807(-1)$ \\
\hline .18877734727 & $.43770365881357(-1)$ & .18489794942753 & $.43491062263223(-1)$ \\
\hline .23532835662211 & $.49272530816407(-1)$ & .23121300154826 & $.49082153228403(-1)$ \\
\hline .28716842664 & $.54312843701643(-1)$ & .28291196019721 & $.54222428661263(-1)$ \\
\hline .34372968768336 & $.58680474177812(-1)$ & .33943548119085 & $.58695944290977(-1)$ \\
\hline .40423850186626 & $.62176153382847(-1)$ & .40001311310945 & $.62297918114936(-1)$ \\
\hline .46773126684306 & $.64620577387348(-1)$ & .46367885693931 & $.64843445707233(-1)$ \\
\hline .53307832255314 & $.65862153426135(-1)$ & .529295142 & $.66175559851254(-1)$ \\
\hline .59901513975232 & $.65783766285597(-1)$ & .59558439532565 & $.66172295277200(-1)$ \\
\hline .66417966475440 & $.64308280886562(-1)$ & .66116704039692 & $.64752458922921(-1)$ \\
\hline .72715443518779 & $.61402547573361(-1)$ & .72460452817603 & $.61879858349955(-1)$ \\
\hline .78651187749469 & $.57079736900636(-1)$ & .78444573473494 & $.57565803663442(-1)$ \\
\hline .84086105709630 & $.51399898611490(-1)$ & .83927495147866 & $.51869769192466(-1)$ \\
\hline .88889408329736 & $.44468713374991(-1)$ & .88775959761734 & $.44898178495567(-1)$ \\
\hline .92943037965232 & $.36434488347266(-1)$ & .92869579596323 & $.36801362500369(-1)$ \\
\hline .96145713651391 & $.27483573132827(-1)$ & .96105005918741 & $.27768870377414(-1)$ \\
\hline .98416462586884 & $.17834924980349(-1)$ & .98399570352129 & $.18023873784161(-1)$ \\
\hline .99697836948100 & $.77447054256862(-2)$ & .99694595867976 & $.78276701954968(-2)$ \\
\hline
\end{tabular}

The first example is concerned with $\lambda_{2 k}=k$ and $\lambda_{2 k+1}=k+1 / 3, k \in \mathbb{N}$. Using the demonstrated algorithm we are able to safely construct generalized Gaussian quadrature rules with 30 points. The corresponding results are presented in Table 4.1 (left). Numbers in parentheses indicate decimal exponents. The presented precision is achieved in six iterations. Figure 4.1 (left) presents the condition number of the matrix $U_{n}$ as a function of the number of nodes $n$ in the generalized Gaussian quadrature rule (1.7). As we can see, the condition number grows as $n^{2}$, which makes the

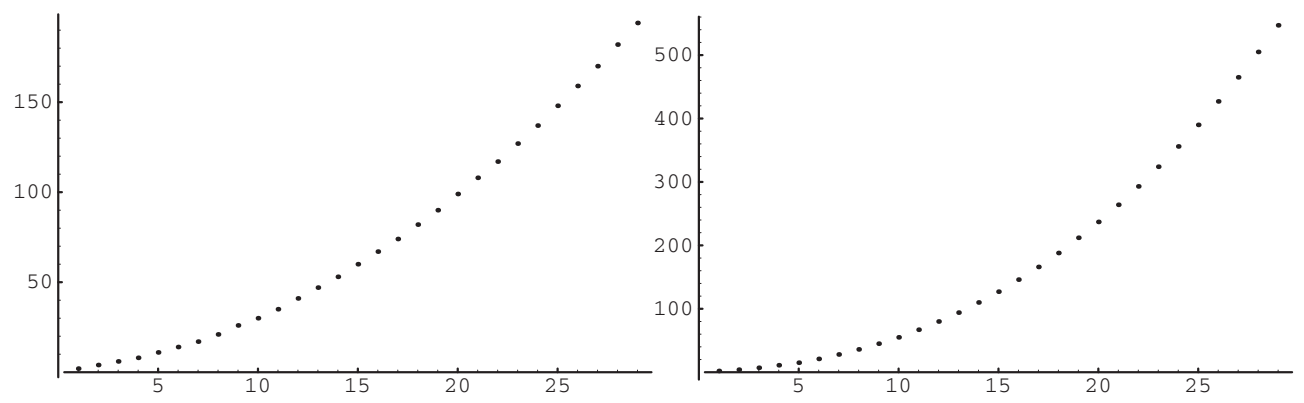

FIG. 4.1. The condition number of the matrices $U_{n}$ (left) and $V_{n} U_{n}^{-1} Y_{n}-Z_{n}$ (right) for $n=2(1) 30$ and $\lambda_{2 k}=k, \lambda_{2 k+1}=k+1 / 3, k \in \mathbb{N}_{0}$. 
TABLE 4.2

Nodes and weights in the generalized Gaussian quadrature rule for $\lambda_{3 k}=\lambda_{3 k+1}=\lambda_{3 k+2}=k$, $k \in \mathbb{N}_{0}$, and 25 points (left) and for $\lambda_{2 k}=k-2 / 3, \lambda_{2 k+1}=k, k \in \mathbb{N}_{0}$, and 30 points (right).

\begin{tabular}{|c|c||c|c|}
\hline Nodes & Weights & Nodes & Weights \\
\hline $.19071312857447(-6)$ & $.89820559783363(-6)$ & $.13087228815024(-5)$ & $.83137155735974(-5)$ \\
$.62344778034991(-5)$ & $.16484108838767(-4)$ & $.54198486279011(-4)$ & $.13031036416570(-3)$ \\
$.55318345803585(-4)$ & $.10072125141830(-3)$ & $.35417216474237(-3)$ & $.52866204357169(-3)$ \\
$.26885580904675(-3)$ & $.37100307224423(-3)$ & $.12534406542994(-2)$ & $.13520067889260(-2)$ \\
$.92211316431026(-3)$ & $.10179277384384(-2)$ & $.32421307701854(-2)$ & $.27261843607855(-2)$ \\
$.25153686640161(-2)$ & $.22982801041688(-2)$ & $.69217972803109(-2)$ & $.47471314476665(-2)$ \\
$.58293105313034(-2)$ & $.45106638074320(-2)$ & $.12972528592304(-1)$ & $.74751509252983(-2)$ \\
$.11948207244089(-1)$ & $.79560124465681(-2)$ & $.22115108819058(-1)$ & $.10930789003913(-1)$ \\
$.22237933600721(-1)$ & $.12887776775257(-1)$ & $.35069941343596(-1)$ & $.15092506726768(-1)$ \\
$.38271779896341(-1)$ & $.19458551169345(-1)$ & $.52514592060155(-1)$ & $.19896260127508(-1)$ \\
$.61704355074579(-1)$ & $.27670975632577(-1)$ & $.75041883938197(-1)$ & $.25237021047441(-1)$ \\
$.94101825972594(-1)$ & $.37340754197965(-1)$ & .10312046455617 & $.30972186656745(-1)$ \\
.13674411641697 & $.48078533727343(-1)$ & .13705967503275 & $.36926743841072(-1)$ \\
.19042046600597 & $.59295303951325(-1)$ & .17698037618878 & $.42899978314872(-1)$ \\
.25524304731076 & $.70233159794501(-1)$ & .22279314304338 & $.48673450869232(-1)$ \\
.33050359479737 & $.80020063322645(-1)$ & .27418493220982 & $.54019907442052(-1)$ \\
.41459502770349 & $.87744065586239(-1)$ & .33061497138402 & $.58712748099728(-1)$ \\
.50501408859338 & $.92539713525291(-1)$ & .39132023098003 & $.62535654329125(-1)$ \\
.59845270059371 & $.93677440023386(-1)$ & .45533043158372 & $.65291965366787(-1)$ \\
.69097602175889 & $.90645885312278(-1)$ & .52149213454311 & $.66813403026136(-1)$ \\
.77827520264508 & $.83217463141567(-1)$ & .58850107398115 & $.66967770271901(-1)$ \\
.85597385719824 & $.71489061053272(-1)$ & .65494153338288 & $.65665290563282(-1)$ \\
.91996040353326 & $.55892462615736(-1)$ & .71933126384264 & $.62863311010376(-1)$ \\
.96671495434044 & $.37173422115917(-1)$ & .78017019720500 & $.58569160378130(-1)$ \\
.99360559333526 & $.16363377320650(-1)$ & .83599103637665 & $.52841030340611(-1)$ \\
& & .88540971524184 & $.45786833033451(-1)$ \\
& & .92717371952416 & $.37561082160968(-1)$ \\
& .96020636853232 & $.28359983739178(-1)$ \\
& & .98364554126283 & $.18415501918852(-1)$ \\
& & .99687876726025 & $.79996620858817(-2)$ \\
\hline
\end{tabular}

construction possible. The condition number of the matrix $V_{n} U_{n}^{-1} Y_{n}-Z_{n}$ is given in Figure 4.1 (right). The presented condition number of the matrix $U_{n}$ suggests that we should be ready to lose certain digits of precision with which we are working. Actually, experiments show that the presented algorithm, in double precision arithmetic (REAL*8), gives exact results with 14 significant digits. Thus, the results presented in Table 4.1 (left) have all digits exact.

Another example is presented in Table 4.1 (right) that is the generalized Gaussian quadrature rule for the sequence $\lambda_{2 k}=\lambda_{2 k+1}=k, k \in \mathbb{N}_{0}$, or for the system of functions $x^{k}, x^{k} \log x, k \in \mathbb{N}_{0}$. In this case, the condition numbers of $U_{n}$, as well as of $V_{n} U_{n}^{-1} Y_{n}-Z_{n}$ (see Figure 4.2), are growing faster than in the previous example. The same behavior we encounter in the example for $\lambda_{3 k}=\lambda_{3 k+1}=\lambda_{3 k+2}=k, k \in \mathbb{N}_{0}$, given in Table 4.2, with condition numbers presented in Figure 4.3. In the latter case, the condition numbers are bigger. This behavior can be understand according to Lemma 3.4. In these two cases the $\Lambda$ sequence is quite different from the $\Lambda$ sequence in the Legendre case. It turns out that, using the presented algorithm in double precision arithmetic $\left(\mathrm{REAL}^{*} 8\right)$, we can safely calculate 14 digits.

In the final example we present an application of our algorithm to a computation of the generalized Gaussian quadrature formula (1.7) in the case when the basis functions are nonintegrable. Consider the system of functions $x^{k-2 / 3} x^{k}, k \in \mathbb{N}_{0}$. For this system of functions our algorithm cannot be applied directly, since the sequence 

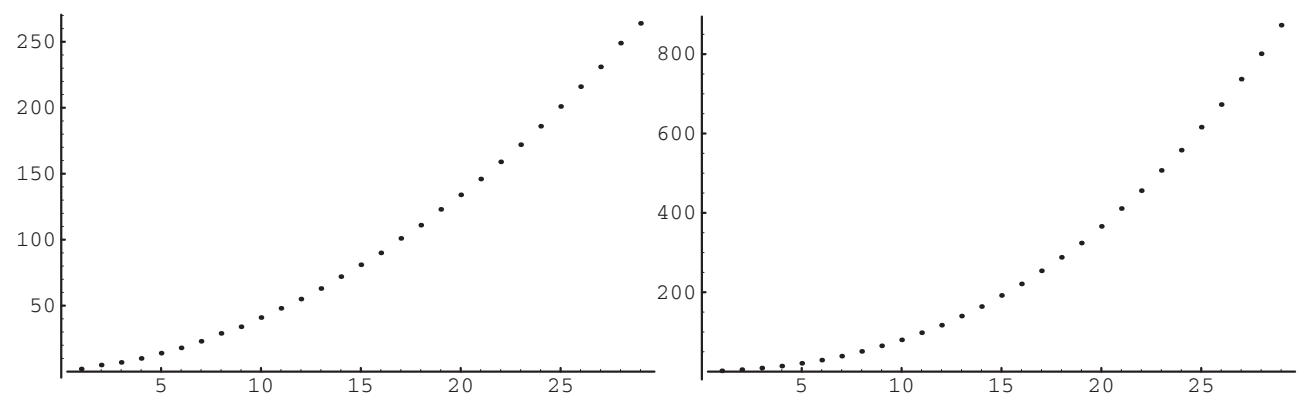

FIG. 4.2. The condition number of the matrices $U_{n}$ and $V_{n} U_{n}^{-1} Y_{n}-Z_{n}$ for $n=2(1) 30$ and $\lambda_{2 k}=\lambda_{2 k+1}=k, k \in \mathbb{N}_{0}$.

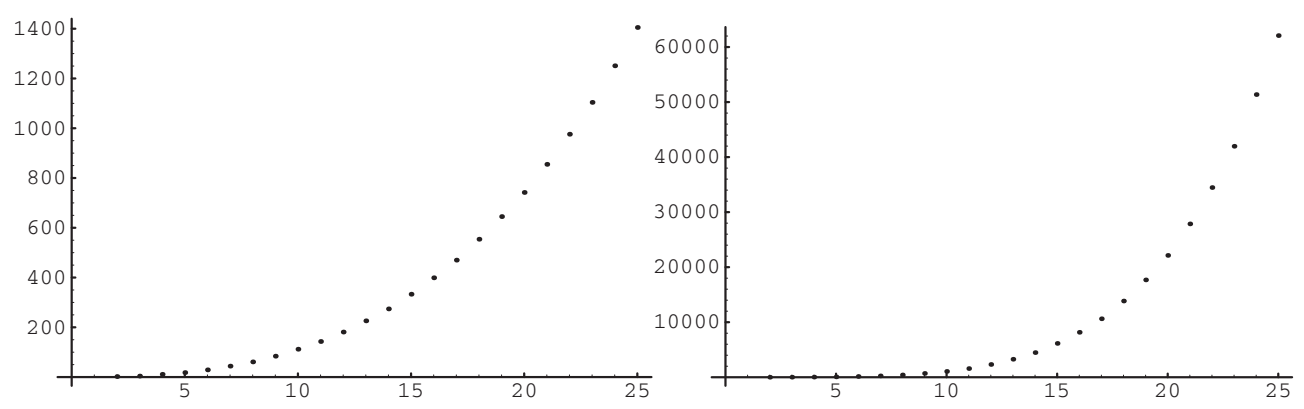

FIG. 4.3. The condition number of the matrices $U_{n}$ and $V_{n} U_{n}^{-1} Y_{n}-Z_{n}$ for $n=2(1) 30$ and $\lambda_{3 k}=\lambda_{3 k+1}=\lambda_{3 k+2}=k, k \in \mathbb{N}_{0}$.

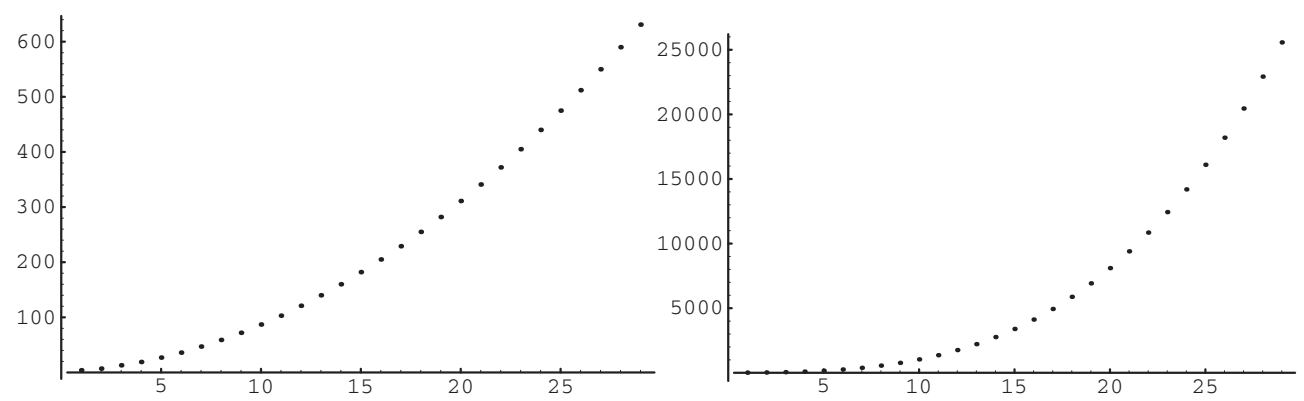

FIG. 4.4. The condition number of the matrices $U_{n}$ and $V_{n} U_{n}^{-1} Y_{n}-Z_{n}$ for $n=2(1) 30$ and $\lambda_{2 k}=k-2 / 3, \lambda_{2 k+1}=k, k \in \mathbb{N}_{0}$.

$\lambda_{2 k}=k-2 / 3, \lambda_{2 k+1}=k, k \in \mathbb{N}_{0}$, does not satisfy the condition $\operatorname{Re}\left(\lambda_{k}\right)>-1 / 2$ and orthogonal Müntz-Legendre polynomials do not exist. However, we can apply Lemma 3.5. For example, we can choose $\beta=2 / 3$ and apply our algorithm for constructing the generalized Gaussian quadrature rule (1.7) for the sequence $\lambda_{2 k}=k-1 / 3$, $\lambda_{2 k+1}=k+1 / 3, k \in \mathbb{N}_{0}$. Using Lemma 3.5, once our algorithm is applied, we can construct the generalized Gaussian quadrature rule for the system of functions $x^{k-2 / 3}$, $x^{k}, k \in \mathbb{N}_{0}$, with respect to the Legendre measure. The results for $n=30$ nodes in the generalized Gaussian quadrature rule (1.7) are presented in Table 4.2 (right). The corresponding condition numbers are given in Figure 4.4. 
TABLE 4.3

Relative errors for the classical Gauss-Legendre quadrature, with $n=100(100) 600$ points, in evaluation of (4.1).

\begin{tabular}{|c|c|c|c|c|c|c|}
\hline$n$ & 100 & 200 & 300 & 400 & 500 & 600 \\
\hline r. err & $1.2(-3)$ & $3.0(-4)$ & $1.3(-4)$ & $7.4(-5)$ & $4.7(-5)$ & $3.3(-5)$ \\
\hline
\end{tabular}

TABLE 4.4

Relative errors for the generalized Gaussian quadrature rule, with $n=5$, 10, and 15 nodes, in evaluation of (4.1), for the sequence $\lambda_{2 k}=\lambda_{2 k+1}=k, k \in \mathbb{N}_{0}$.

\begin{tabular}{|c|c|c|c|}
\hline$n$ & 5 & 10 & 15 \\
\hline r. err & $9.0(-7)$ & $1.3(-15)$ & m.p. \\
\hline
\end{tabular}

At the end of this section we present an application of the derived quadrature formulae. We consider the integral

$$
\int_{0}^{1} J_{0}(x)(1+\log x) d x=-0.0531080375895118730468486186978172 \ldots,
$$

where $J_{0}(x)$ is the Bessel function. Table 4.3 lists relative errors for the classical Gauss-Legendre quadrature rule, shifted to the interval $(0,1)$, applied to the previous integral. This is an example of the well-known saturation phenomenon (see [20]). However, using the generalized Gaussian quadrature rule derived for the sequence $\lambda_{2 k}=\lambda_{2 k+1}=k, k \in \mathbb{N}_{0}$, the machine precision (m.p. $\approx 2.26(-16)$ ) can be achieved with 15 nodes, as presented in Table 4.4.

\section{REFERENCES}

[1] I. V. Andronov, Application of orthogonal polynomials in the boundary-contact value problems, in Proceeding VIII Simposium Sobre Polinomios Ortogonales y Sus Aplicaciones (Sevilla, Sept. 22-26, 1997), Book of Abstracts, Facultad de Matemáticas, Universidad de Sevilla, 1997, p. 32.

[2] G. V. Badalyan, Generalization of Legendre polynomials and some of their applications, Akad. Nauk Armyan. SSR. Izv. Fiz.-Mat. Estest. Tehn. Nauk, 8 (1955), pp. 1-28; 9 (1956), pp. 322 (in Russian; Armenian summary).

[3] P. Borwein, T. ERdÉlyi, AND J. Zhang, Müntz systems and orthogonal Müntz-Legendre polynomials, Trans. Amer. Math. Soc., 342 (1994), pp. 523-542.

[4] P. Borwein And T. Erdélyi, Polynomials and Polynomial Inequalities, Graduate Texts in Math. 161, Springer-Verlag, New York, 1995.

[5] T. S. Chinara, An Introduction to Orthogonal Polynomials, Gordon and Breach, New York, 1978.

[6] W. Gautschi, A survey of Gauss-Christoffel quadrature formulae, in E. B. Christoffel. The Influence of His Work on Mathematics and the Physical Sciences, P. L. Butzer and F. Fehér, eds., Birkhäuser, Basel, 1981, pp. 72-147.

[7] W. Gautschi, Algorithm 726: ORTHPOL - a package of routines for generating orthogonal polynomials and Gauss-type quadrature rules, ACM Trans. Math. Software, 20 (1994), pp. 21-62.

[8] W. Gautschi, Orthogonal Polynomials, Computation, and Approximation, Oxford University Press, New York, 2004.

[9] G. Golub And J. H. Welsch, Calculation of Gauss quadrature rules, Math. Comp., 44 (1969), pp. $221-230$.

[10] A. S. Householder, The Theory of Matrices in Numerical Analysis, Dover, New York, 1975.

[11] C. G. HARRIS AND W. A. B. Evans, Extension of numerical quadrature formulae to cater for end point singular behaviours over finite intervals, Internat. J. Comput. Math., 6 (1977), pp. 219-227.

[12] S. Karlin And W. J. Studden, Tchebycheff Systems with Applications in Analysis and Statistics, Pure and Appl. Math. XV, Wiley Interscience, New York, 1966. 
[13] J. Ma, V. Rokhlin, And S. Wandzura, Generalized Gaussian quadrature rules for systems of arbitrary functions, SIAM J. Numer. Anal., 33 (1996), pp. 971-996.

[14] A. A. Markov, Selected Papers on Continued Fractions and the Theory of Functions Deviating Least from Zero, OGIZ, Moscow, Leningrad, 1948 (in Russian).

[15] P. C. McCarthy, J. E. Sayre, and B. L. R. Shawyer, Generalized Legendre polynomials, J. Math. Anal. Appl., 177 (1993), pp. 530-537.

[16] G. V. Milovanović, Numerical calculation of integrals involving oscillatory and singular kernels and some applications of quadratures, Comput. Math. Appl., 36 (1998), pp. 19-39.

[17] G. V. Milovanović, Müntz orthogonal polynomials and their numerical evaluation, in Applications and Computation of Orthogonal Polynomials, Internat. Ser. Numer. Math. 131, W. Gautschi, G. H. Golub, and G. Opfer, eds., Birkhäuser, Basel, 1999, pp. 179-194.

[18] G. V. Milovanović, Numerical Analysis, Part I, 3rd ed., Naučna Knjiga, Belgrade, 1991 (in Serbo-Croatian)

[19] G. V. Milovanović and A. S. Cvetković, Note on a construction of weights in Gauss-type quadrature rule, Facta Univ. Ser. Math. Inform., 15 (2000), pp. 69-83.

[20] G. V. Milovanović and A. S. CvetKović, Numerical integration of functions with logarithmic end point singularity, Facta Univ. Ser. Math. Inform., 17 (2002), pp. 57-74.

[21] P. G. Nevai, Orthogonal Polynomials, Mem. Amer. Math. Soc. 18, AMS, Providence, RI, 1979.

[22] T. J. Stieltjes, Sur une généralisation de la théorie des quadratures mécaniques, C.R. Acad. Sci. Paris, 99 (1884), pp. 850-851.

[23] J. A. Sнонат, On a certain formula of mechanical quadratures with non-equidistant ordinates, Trans. Amer. Math. Soc., 31 (1929), pp. 448-463.

[24] G. Szegö, Orthogonal Polynomials, 4th ed., Amer. Math. Soc. Colloq. Publ. 23, AMS, Providence, RI, 1975.

[25] A. K. Taslakyan, Some properties of Legendre quasipolynomials with respect to a Müntz system, in Mathematics, vol. 2, Èrevan University, Èrevan, 1984, pp. 179-189 (Russian, Armenian summary).

[26] C. W. Ueberhuber, Numerical Computation 2: Methods, Software, and Analysis, SpringerVerlag, Berlin, 1997.

[27] W. Van Assche, Approximation theory and analytic number theory, Special Functions and Differential Equations (Madras, 1997), Allied Publ., New Delhi, 1998, pp. 336-355.

[28] N. YARVin And V. Rokhlin, Generalized Gaussian quadratures and singular value decompositions of integral operators, SIAM J. Sci. Comput., 20 (1998), pp. 699-718. 